\title{
Hyperlipidemia as a predictor of physical functioning for stroke
}

\author{
Jae-hong Sim ${ }^{a}$, Sujin Hwang ${ }^{b}$, Chiang-soon Song ${ }^{c}$ \\ ${ }^{a}$ Department of Physical Therapy, Graduate School of Health and Welfare, Baekseok University, Seoul, Republic of Korea \\ ${ }^{b}$ Department of Physical Therapy, Division of Health Science, Baekseok University, Cheonan, Republic of Korea \\ 'Department of Occupational Therapy, College of Health Science, Chosun University, Gwangju, Republic of Korea
}

Objective: Elevated cholesterol levels contribute to changes of the arterial endothelial permeability. Hyperlipidemia promotes atherosclerosis and is associated with an increased risk of stroke incidence. The purpose of this study was to investigate the effects of having a history of hyperlipidemia prior to a stroke incidence on postural balance, anticipatory dynamic postural control, gait endurance and gait performance in individuals with hemiparetic stroke.

Design: Cross-sectional study.

Methods: Fifty-two adults who were diagnosed with stroke 6 months ago or more were enrolled in this study. They were divided into two different groups according to hyperlipidemia history before stroke. All participants were assessed with the Activities-specific into Balance Confidence (ABC) scale, Berg Balance scale (BBS), Dynamic Gait Index (DGI), Timed Up and Go test (TUG), and the 6-minute walk test (6MWT). An independent t-test was used to analyze the difference between the hyperlipidemia group and non-hyperlipidemia group.

Results: After analysis, the BBS, TUG, and 6MWT scores were significantly different between the hyperlipidemia and non-hyperlipidemia group, but not the ABC and DGI scores.

Conclusions: The results of this study show that having a history of hyperlipidemia before stroke affects static and dynamic postural balance performance, anticipatory dynamic postural balance, and gait endurance in individuals with chronic hemiparetic stroke. Based on the results of this study, we also suggest treatment for hyperlipidemia should be implemented throughout the therapeutic interventions, such as pharmacological or exercise programs, in order to restore the physical function of stroke survivors.

Key Words: Gait, Hyperlipidemia, Postural balance, Stroke

\section{Introduction}

Stroke is a major global public health burden in Organization for Economic Cooperation and Development (OECD) countries and is the leading cause of permanent disabilities among adults caused by a disruption of the blood flow due to blood vessel injuries in the brain. Stroke and other cerebrovascular diseases accounted for approximately $7 \%$ of all deaths in OECD countries in 2013 [1]. Clinically, stroke survivors have a variety of focal deficits according to their brain injury areas including changes in the level of consciousness and impairments of sensory, motor, cognitive, perceptual, and language functions [2]. Stroke survivors may suffer long-term or permanent disabilities caused by brain injury if they do not receive proper physical and cognitive training throughout rehabilitation programs [3].

A major contributory factor in stroke is atherosclerosis, which it is characterized by plaque formation with an accumulation of lipids, fibrin, complex carbohydrates, and calcium deposits on the arterial walls that leads to progressive narrowing of blood vessels $[2,4]$. Atherosclerosis can produce a thrombus, embolism, or conditions that yield to low

Received: 24 May, 2018 Revised: 10 June, 2018 Accepted: 10 June, 2018 
systemic perfusion pressures in the brain vessels, resulting in ischemic strokes, and small blood vessels are weakened by atherosclerosis, which may produce an aneurysm and bring about cerebral or nontraumatic spontaneous hemorrhage $[2,4]$. Therefore, atherosclerosis management is important in order to reduce the incidence of stroke within the public health.

One of major causes of atherosclerosis is hyperlipidemia caused by an excessive uptake of a high-cholesterol diet leading to high levels of blood lipids, especially cholesterol $[5,6]$. Elevated cholesterol levels contribute to changes in the arterial endothelial permeability since hyperlipidemia promotes atherosclerosis and is associated with an increased risk of stroke incidence [6]. Recently, one study investigated the influences of hyperlipidemia on the early outcome of stroke by estimating the odds ratio for the sequelae requiring care and hazard ratio for death [7]. They suggested that the outcome was favorable for patients with a history of hyperlipidemia in terms of both the remaining sequelae and hazard ratio for death. Factors that increase the incidence of the disease could have an influence on the severity of the disease in a favorable way [7]. However, they did not investigate the effects of having a history of hyperlipidemia before stroke on physical function for stroke survivors, and there is insufficient information on the effects of hyperlipidemia on physical activity performance ability following stroke, even though hyperlipidemia promotes atherosclerosis and affects both the brain and heart.

The purpose of this study was to evaluate the effects of having a history of hyperlipidemia on physical activities in persons with chronic hemiparetic stroke. We divided the subjects into two groups, including the hyperlipidemia group and non-hyperlipidemia group, to evaluate the effects of having a history of hyperlipidemia on physical activities. The hypothesis of this study was that having a hyperlipidemia history will affect the performance of physical activities, such as postural balance and gait performance in persons with chronic hemiparetic stroke.

\section{Methods}

\section{Participants}

Fifty-two persons who had been diagnosed with stroke 6 or more months ago participated in this study. The participants had been informed about the purpose and procedures of the study and had voluntarily agreed to participate. The inclusion criteria for the participants were as follows: (a) first stroke with an onset of 6 months or more, (b) no severe visuoperceptual impairment, (c) no severe somatosensory impairment, (d) the ability to maintain a sitting position in a chair for longer than 20 minutes, (e) the ability to walk independently with or without an assistive device for 10 meters, (f) the absence of any orthopedic disorders that could potentially affect the results of the study, and $(\mathrm{g})$ the absence of neurological disorders except for stroke. The study protocol was approval by the Baekseok University Human Research Ethics Committee (Approval No. BUIRB201706-HR-008), and written informed consent was obtained from all participants. Table 1 shows the common characteristics of all the participants in this study.

\section{Procedures}

This was a cross-sectional study to observe for any differences in physical function, such as postural balance efficacy

Table 1. Common characteristics of participants

$(\mathrm{N}=52)$

\begin{tabular}{lccrc}
\multicolumn{1}{c}{ Variable } & $\begin{array}{c}\text { Non-hyperlipidemia } \\
\text { group }(\mathrm{n}=37)\end{array}$ & $\begin{array}{c}\text { Hyperlipidemia } \\
\text { group (n=15) }\end{array}$ & t & $p$-value \\
\hline Age (y) & $60.14(10.76)$ & $60.93(12.60)$ & -0.231 & 0.819 \\
Height (cm) & $164.08(6.80)$ & $166.27(7.67)$ & -1.012 & 0.316 \\
Weight (kg) & $67.31(14.76)$ & $63.84(7.94)$ & 0.858 & 0.395 \\
Drug intake (n) & $6.95(2.29)$ & $5.87(2.39)$ & 1.524 & 0.134 \\
Post-disease duration (mo) & $27.11(26.20)$ & $28.33(24.36)$ & -0.156 & 0.877 \\
MMSE (score) & $27.43(2.21)$ & $26.07(2.28)$ & 2.003 & 0.051 \\
Sex (male/female) & $26 / 11$ & $9 / 6$ & - & - \\
Etiology (infarction/hemorrhage) & $27 / 10$ & $11 / 4$ & - & - \\
Brunnstrom stage (4/5/6) & $9 / 10 / 18$ & $6 / 7 / 2$ & - & - \\
Paretic side (right/left) & $24 / 13$ & $9 / 6$ & - & - \\
\hline
\end{tabular}

Values are presented as mean (SD) or number only.

MMSE: Mini-Mental Status Examination. 
and performance, anticipatory dynamic postural balance, and gait performance and endurance between hyperlipidemia and non-hyperlipidemia groups. First, this study divided all participants into either the hyperlipidemia or non-hyperlipidemia group according to their medical history before stroke. After applying the inclusion and exclusion criteria, 52 participants were eligible to participate in the study (37 hyperlipidemia group and 15 non-hyperlipidemia group). All participants conducted four performance-based clinical measures including the Berg Balance scale (BBS), Dynamic Gait Index (DGI), Timed Up and Go test (TUG), 6-minute walk test (6MWT), and one interviewbased clinical measure, the Activities-Specific Balance Confidence $(\mathrm{ABC})$ scale. All clinical measures were assessed in a calm and well-organized therapy room by two rehabilitative therapists who had a clinical experience for 10 years or more.

\section{Outcome measures}

The $\mathrm{ABC}$ scale is an interview-based questionnaire developed to measure the aspect of the psychological impact of postural balance impairment or falls risk. The tool is based on the self-efficacy theory reported by Tinetti et al. [8]. It indicates the patient's level of confidence in doing the activity without losing postural balance or becoming unsteady from choosing one of the percentage points on the scale from $0 \%$ (no confidence) to $100 \%$ (completely confident). An average percentage for each of the 16 items is calculated [9]. The cut-off score for the $\mathrm{ABC}$ is $67 \%$, and was reported to have a sensitivity of $84 \%$ and specificity of $87 \%$ in a cross-sectional study on community-dwelling elderly people [10]. The tool has been shown to have excellent reliability and validity $[11,12]$.

The BBS is a performance-based, postural balance measurement and was developed to measure postural balance among elderly persons with postural impairments by assessing their performance in functional tasks [13]. The BBS is used to objectively determine a patient's ability to safely balance during a series of predetermined tasks. It is a 14-item list with each item consisting of a 5-point ordinal scale ranging from 0 (the lowest level of function) to 4 (the highest level of function) and takes approximately 20 minutes to complete [13]. This tool has been reported to have excellent reliability and validity [14].

The DGI was developed as a clinical tool to assess walking, postural balance, and risk of falls. The tool evaluates the usual steady-state walking and more challenging dynamic gait performances. Eight functional gait tests are performed by the patient [2]. The tool is graded on a four-point ordinal scale, ranging from 0 (the lowest level of function) to 3 (the highest level of function), with 24 as the total possible individual score. Scores of 19 or less have been related to increase risk of falls and scores of 22 or more have been related to safe ambulation. Equipment needed is a shoebox, cones, stairs, and a walkway (20 feet length, 15 -inch wide). The completion time of the tool is appropriately 15 minutes [2]. The DGI shows sufficient validity, responsiveness, and reliability for the assessment of gait performance in persons with stroke undergoing rehabilitation [15].

The TUG was developed to determine fall risk and to measure the progress of postural balance, sit-to-stand, and walking designed for elderly persons initially. Materials needed are a chair with armrests, stop watch, and tape to mark 3 meters [16]. The client starts in a seated position on a chair with armrests, stands up upon the therapist's command, "Go," walks 3 meters, turns around, walks back to the chair and sits down. The client is allowed to use an assistive device during the assessment. The participant is classified as high-risk for falling if he or she takes 14 seconds to complete the test [16]. The TUG has been reported to have excellent reliability and validity [17].

The 6MWT is a performance-based measure and a submaximal exercise test of functional exercise capacity and gait tolerance developed in 1963 by Balke [18]. The tool measures the distance covered over a time of 6 minutes as the outcome in order to be able to compare changes in performance capacity. Equipment required is a stopwatch, measuring wheel to measure distance covered, 30-meter stretch of unimpeded walkway, pulse oximeter for measuring heart rate and $\mathrm{SpO} 2$, and the Borg Dyspnea Scale. First of all, the evaluator places cones at either ends of the 30-meter stretch as turning points and has a chair set up on either side and halfway along the walkway. The participant is to walk as far as possible for 6 minutes. If he or she become short of breath or become exhausted, he or she is permitted to slow down, stop, and to rest as necessary [19]. The 6MWT has been reported to have excellent reliability and validity $[20,21]$.

\section{Statistical analysis}

In order to analyze the general characteristics of the participants, descriptive statistics was used, and the frequency, percentage, mean and standard deviation values were obtained. The independent variable is the history of hyper- 
lipidemia before stroke, and the dependent variables are postural balance confidence and performance, and gait performance and endurance. The independent t-test was used to compare the dependent variables between the two groups. In order to compare postural balance and gait performance between the two groups, a regression analysis was used. Data collection was analyzed using the PASW Statistics ver. 18.0 (IBM Co., Armonk, NY, USA), with a significant level set at $p<0.05$.

\section{Results}

This study analyzed postural balance and gait performance with hyperlipidemia or without hyperlipidemia history for individuals with chronic hemiparetic stroke (Table 2). The ABC, BBS, and DGI scores were higher in the non-hyperlipidemia group compared to those in the hyperlipidemia group. The TUG scores were faster in the non-hyperlipidemia group compared to the hyperlipidemia group. The distance of the 6MWT was greater in the non-hyperlipidemia group compared to the hyperlipidemia group. Although the BBS, TUG, and 6MWT were significantly different between the two groups, the $\mathrm{ABC}$ and DGI scores were not significantly different (Table 2). The BBS, TUG, and 6MWT were significantly different between the hyperlipidemia and non-hyperlipidemia group (Table 3).

\section{Discussion}

This study examined the postural balance confidence and performance, and gait endurance and performance in persons with chronic hemiparetic stroke who had a history or without a history of hyperlipidemia prior stroke. The main results of this study were as follows: poostural balance, anticipatory dynamic balance, and gait endurance was superior in the non-hyperlipidemia history compared to the hyperlipidemia history group.

Previous studies have reported that appropriately half of ischemic stroke patients exhibit hyperlipidemia, and patients with hyperlipidemia in turn have an elevated stroke mortality [22-24]. Hermann and Chopp [22] reported that impaired angiogenesis in stroke survivors with hypercholesterolemia parallels disturbances in synaptic plasticity, and lipid-lowering drugs are widely prescribed for individuals with stroke as secondary stroke prevention. Horenstein et al. [24] evaluated the risk of death caused by stroke, age, and cholesterol rate in 24.343 women with no previous history of cardiovascular diseases. They reported that cholesterol levels is a risk factor for non-hemorrhagic stroke death in women aged 55 years or less and is more powerfully associated with mortality in black women aged 55 years or less than in white women, and that cholesterol level is important in addition to established risk factors for predicting stroke mortality in young women and to guide prevention strategies. Zechariah et al. [25] reported that hyperlipidemia de-

Table 2. Postural balance confidence and physical functioning and gait endurance and performance

$(\mathrm{N}=52)$

\begin{tabular}{lccrc}
\hline \multicolumn{1}{c}{ Variable } & Non-hyperlipidemia group $(\mathrm{n}=37)$ & Hyperlipidemia group $(\mathrm{n}=15)$ & $\mathrm{t}$ & $p$-value \\
\hline ABC (score) & $58.44(27.69)$ & $46.59(23.65)$ & 1.455 & 0.152 \\
BBS (score) & $45.41(9.8)$ & $39.53(9.20)$ & 2.046 & 0.049 \\
DGI (score) & $16.54(5.75)$ & $14.07(5.18)$ & 1.511 & 0.142 \\
TUG (s) & $25.60(20.73)$ & $44.29(29.03)$ & -2.615 & 0.012 \\
6MWT (m) & $223.96(118.47)$ & $150.45(86.20)$ & 2.175 & 0.034 \\
\hline
\end{tabular}

Values are presented as mean (SD).

ABC: Activities-Specific Balance Confidence, BBS: Berg Balance scale, DGI: Dynamic Gait Index, TUG: Timed Up and Go test, 6MWT: 6-minute walk test.

Table 3. Regression between hyperlipidemia and postural balance, and gait performance

$(\mathrm{N}=52)$

\begin{tabular}{lrrrrr}
\hline Variable & B & SE & $\beta$ & t & $p$-value \\
\hline BBS & -5.872 & 2.950 & -0.271 & -1.991 & 0.049 \\
TUG & 18.693 & 7.149 & 0.347 & 2.615 & 0.012 \\
6MWT & -73.503 & 33.789 & -0.294 & -2.175 & 0.034 \\
\hline
\end{tabular}

BBS: Berg Balance scale, TUG: Timed Up and Go test, 6MWT: 6-minute walk test. 
creases vascular endothelial growth factor-induced angiogenesis, damages blood flow in the brain, and interrupts stroke recovery via decreased pericyte coverage of brain endothelial cells. They also reported that the compromised vessel formation and hemodynamics question the concept of therapeutic angiogenesis in ischemic stroke where hyperlipidemia is highly prevalent [25].

This study evaluated the effects of having a history of hyperlipidemia before stroke on physical function, such as postural balance and gait performance, in individuals with stroke. The participants chronic hemiparetic stroke survivors and received physical therapeutic interventions in rehabilitation centers since they had been diagnosed with stroke. This study divided the participants into two different groups according to their medical history of hyperlipidemia prior to stroke. The results of this study suggest that hyperlipidemia disturbs restorative recovery of postural and anticipatory dynamic postural balance as well as gait endurance following stroke. Oza et al. [26] reported that hyperlipidemia should be well-managed through pharmacological interventions, such as aspirin, to prevent recurrent ischemic stroke, although they did not analyze the same dependent variables of this study. Gan et al. [27] reported that population-based public health intervention programs and policies targeting hyperlipidemia and hypertension control and encouragement of physical activity should be high prioritized for middle-aged and older adults in order to prevent stroke.

This study was a cross-sectional observational study design, which examined the physical function between those with or without hyperlipidemia history before stroke. This study suggests future studies should further investigate the therapeutic effects of hyperlipidemia management on physical function after stroke. Based on the results of this study, having a history of hyperlipidemia showed a disturbance on postural balance, anticipatory dynamic balance, and gait endurance for individuals with chronic hemiparetic stroke.

\section{Conflict of Interest}

The authors declared no potential conflicts of interest with respect to the authorship and/or publication of this article.

\section{References}

1. GBD 2013 DALYs and HALE Collaborators, Murray CJ, Barber
RM, Foreman KJ, Abbasoglu Ozgoren A, Abd-Allah F, et al. Global, regional, and national disability-adjusted life years (DALYs) for 306 diseases and injuries and healthy life expectancy (HALE) for 188 countries, 1990-2013: quantifying the epidemiological transition. Lancet 2015;386:2145-91.

2. O'Sullivan SB, Schmitz TJ, Fulk GD. Physical rehabilitation. 6th ed. Philadelphia: F.A. Davis Co.; 2014.

3. Ilunga Tshiswaka D, Seals SR, Raghavan P. Correlates of physical function among stroke survivors: an examination of the 2015 BRFSS. Public Health 2018;155:17-22.

4. Umphred DA. Umphred's neurological rehabilitation. 6th ed. St. Louis, Mo.: Elsevier/Mosby; 2013.

5. Wada S, Koga M, Toyoda K, Minematsu K, Yasaka M, Nagai Y, et al. Factors associated with intima-media complex thickness of the common carotid artery in Japanese noncardioembolic stroke patients with hyperlipidemia: The J-STARS echo study. J Atheroscler Thromb 2018;25:359-73.

6. Bergheanu SC, Bodde MC, Jukema JW. Pathophysiology and treatment of atherosclerosis : current view and future perspective on lipoprotein modification treatment. Neth Heart J 2017;25: 231-42.

7. Shigematsu K, Watanabe Y, Nakano H; Kyoto Stroke Registry Committee. Influences of hyperlipidemia history on stroke outcome; a retrospective cohort study based on the Kyoto Stroke Registry. BMC Neurol 2015;15:44.

8. Tinetti ME, Richman D, Powell L. Falls efficacy as a measure of fear of falling. J Gerontol 1990;45:P239-43.

9. Myers AM, Fletcher PC, Myers AH, Sherk W. Discriminative and evaluative properties of the activities-specific balance confidence (ABC) scale. J Gerontol A Biol Sci Med Sci 1998;53: M287-94.

10. Lajoie Y, Gallagher SP. Predicting falls within the elderly community: comparison of postural sway, reaction time, the Berg balance scale and the Activities-specific Balance Confidence (ABC) scale for comparing fallers and non-fallers. Arch Gerontol Geriatr 2004;38:11-26.

11. Powell LE, Myers AM. The Activities-specific Balance Confidence (ABC) scale. J Gerontol A Biol Sci Med Sci 1995;50A: M28-34.

12. Nilsagård YE, Forsberg A. Practicability and sensitivity to change of the activities-specific balance confidence scale and 12-item Walking Scale for stroke. Top Stroke Rehabil 2012;19: 13-22.

13. Berg K, Wood-Dauphinee S, Williams JI. The Balance Scale: reliability assessment with elderly residents and patients with an acute stroke. Scand J Rehabil Med 1995;27:27-36.

14. Blum L, Korner-Bitensky N. Usefulness of the Berg Balance Scale in stroke rehabilitation: a systematic review. Phys Ther 2008;88:559-66.

15. Jonsdottir J, Cattaneo D. Reliability and validity of the dynamic gait index in persons with chronic stroke. Arch Phys Med Rehabil 2007;88:1410-5.

16. Shumway-Cook A, Brauer S, Woollacott M. Predicting the probability for falls in community-dwelling older adults using the Timed Up \& Go Test. Phys Ther 2000;80:896-903.

17. Bonnyaud C, Pradon D, Zory R, Bensmail D, Vuillerme N, Roche N. Gait parameters predicted by Timed Up and Go performance in stroke patients. NeuroRehabilitation 2015;36:73- 
80.

18. Balke B. A simple field test for the assessment of physical fitness. Rep 63-6. Rep Civ Aeromed Res Inst US 1963;1-8.

19. Ries JD, Echternach JL, Nof L, Gagnon Blodgett M. Test-retest reliability and minimal detectable change scores for the timed "up \& go" test, the six-minute walk test, and gait speed in people with Alzheimer disease. Phys Ther 2009;89:569-79.

20. Eng JJ, Dawson AS, Chu KS. Submaximal exercise in persons with stroke: test-retest reliability and concurrent validity with maximal oxygen consumption. Arch Phys Med Rehabil 2004; 85:113-8.

21. Fulk GD, Echternach JL. Test-retest reliability and minimal detectable change of gait speed in individuals undergoing rehabilitation after stroke. J Neurol Phys Ther 2008;32:8-13.

22. Hermann DM, Chopp M. Promoting brain remodelling and plasticity for stroke recovery: therapeutic promise and potential pitfalls of clinical translation. Lancet Neurol 2012;11:369-80.
23. Sacco RL, Diener HC, Yusuf S, Cotton D, Ounpuu S, Lawton WA, et al. Aspirin and extended-release dipyridamole versus clopidogrel for recurrent stroke. N Engl J Med 2008;359:1238-51.

24. Horenstein RB, Smith DE, Mosca L. Cholesterol predicts stroke mortality in the Women's Pooling Project. Stroke 2002;33:1863-8.

25. Zechariah A, ElAli A, Hagemann N, Jin F, Doeppner TR, Helfrich I, et al. Hyperlipidemia attenuates vascular endothelial growth factor-induced angiogenesis, impairs cerebral blood flow, and disturbs stroke recovery via decreased pericyte coverage of brain endothelial cells. Arterioscler Thromb Vasc Biol 2013;33:1561-7.

26. Oza R, Rundell K, Garcellano M. Recurrent ischemic stroke: strategies for prevention. Am Fam Physician 2017;96:436-40.

27. Gan Y, Wu J, Zhang S, Li L, Yin X, Gong Y, et al. Prevalence and risk factors associated with stroke in middle-aged and older Chinese: A community-based cross-sectional study. Sci Rep 2017;7:9501. 